\title{
Modeling of Localized Lifetime Tailoring in Silicon Devices
}

\author{
P. Hazdra and J. Vobecký
}

\author{
Department of Microelectronics, Czech Technical University of Prague \\ Technická 2, CS-16627 Praha 6, CZECH REPUBLIC
}

\begin{abstract}
A new accurate method for simulation of the device that is subjected to low-dose high-energy ion irradiation (hydrogen, helium) is compared with the ordinary simulation technique which utilizes a structured lifetime profile as an input into SRH recombination model. The novel method, involving ion-implantation process simulator, expert system, and multilevel recombination model is described. Spatial distribution of the minority carrier lifetime of irradiated device is drawn for different injection levels. Simulated trade-off between forward voltage drop and reverse recovery time is presented for both the hydrogen and helium irradiation.
\end{abstract}

\section{Introduction}

Low-dose high-energy ion irradiation is emerging as a powerful tool for local lifetime control in silicon power devices. Light ions, e.g. hydrogen or helium, with energies of several $\mathrm{MeV}$ are used to produce vacancy-related point defects mostly appearing towards the end of the ion range. Since these defects act as recombination centers and carrier traps, the reduction of minority carrier lifetime takes place. Consequently, localized lifetime profile may be optimized, choosing appropriate ion type, irradiation energy and dose, and annealing temperature in order to reach a superior trade-off curve of forward voltage drop versus turn-off time or reverse recovery time. Up to this time, an optimization of irradiation-induced lifetime profiles has been based mostly on the principle of trial-and-error experimentation due to unreliable results of device simulations. This is because the simulators are using only a structured lifetime profile $[1,2]$, a simple recombination model which consider only a single ideal recombination/ generation centre, and there is no connection between the parameters of irradiation and lifetime. However, it is possible to fit some way the profile of the lifetimes $\tau_{\mathrm{n} 0}$ and $\tau_{\mathrm{p} 0}$ for SRH model in order to get a better agreement with experimental results [6], but it is a rather hopeless activity, since the

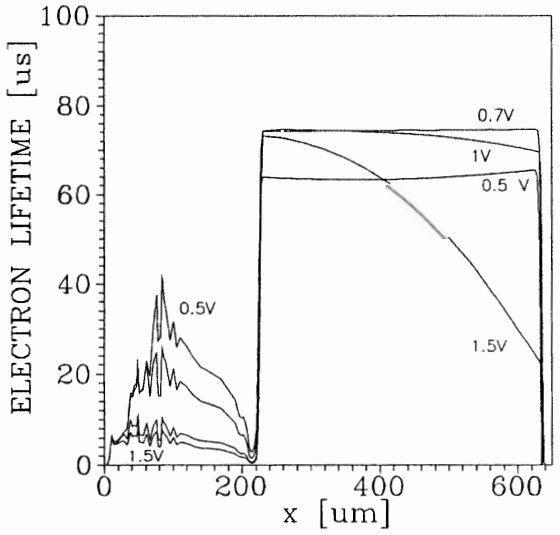

Fig.1: Spatial distribution of electron lifetime $\tau_{\mathrm{n}}=\Delta \mathrm{n} / \mathrm{R}_{\mathrm{n}}$ in diode $0.5,0.7,1$, and $1.5 \mathrm{~V} \mathrm{dc}$ (Irradiated by hydrogen, $5 \mathrm{MeV} / 5 \times 10^{10} \mathrm{~cm}^{-2}$ ) 
actual lifetime profile is very complicated, hard to be accurately measured with desirable spatial resolution, and is bias dependent. It is documented by Fig. 1 which shows the simulated lifetime profile calculated by our new method for a long $\mathrm{P}^{+} \mathrm{PNN}^{+}$power diode to be used throughout the remainder of the paper. The irradiated device volume is located within $0<\mathrm{x}<215 \mu \mathrm{m}$ (the defect maximum is at $\mathrm{x} \doteq 215 \mu \mathrm{m}$ ). During the transient simulation, e.g. reverse recovery process, the profile is even more complicated, especially in the area of desaturated $p-n$ junction. All these circumstances motivated us to developed an exact simulation system to be further described.

\section{The simulation system and its application}

The simulation system (see the Flow Chart) is based on the integration of simulation procedures that simulate the processes of primary and secondary defect generation during irradiation and their influence on electrical properties of irradiated device. Simulation of primary and secondary defect reactions is replaced by an expert system based on an extensive set of DLTS measurements performed on irradiated silicon [3,4] which takes into account technological parameters such as target type (CZ, FZ, resistivity), type of ion projectile, dose, dose rate, irradiation temperature, temperature and time of annealing. It assigns the appropriate deep level to each defect created assuming the fact that the secondary defects are mostly vacancy-related and their number increases linearly with the dose within the interval under study $[3,4]$. The parameters of deep levels (emission and capture rates, charge state and concentration profile) enter into 1-D device simulator [7] which includes improved models of thermal generation / recombination based on SRH statistics and the complete solution of trap-dynamic equations necessary for detailed description of charge flow through complex structure of traps/recombination centres appearing within silicon bandgap after irradiation. An output of the device simulator provides important linkage of the process and electrical characteristics that have been up to this time available only via experiment. Figs. 2 and 3 show the simulated trade-off between voltage

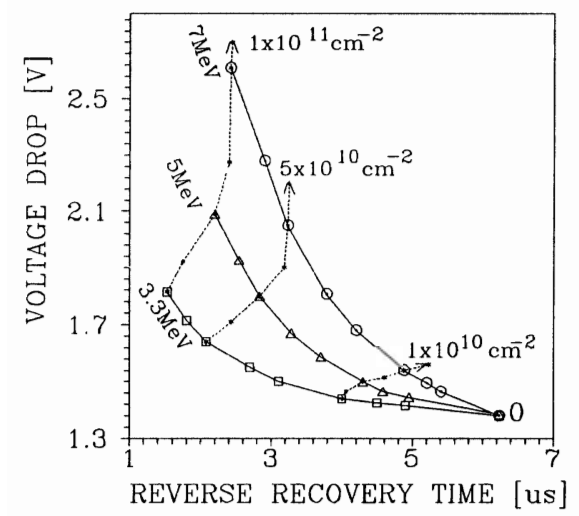

Fig.2: Forward voltage drop-reverse recovery time trade-off for hydrogen (DUT from Figs. 1, 3,4,5), $t_{r r}:-20 \mathrm{~V} / 5 \Omega / 25 \mu \mathrm{H}$ from ON-state

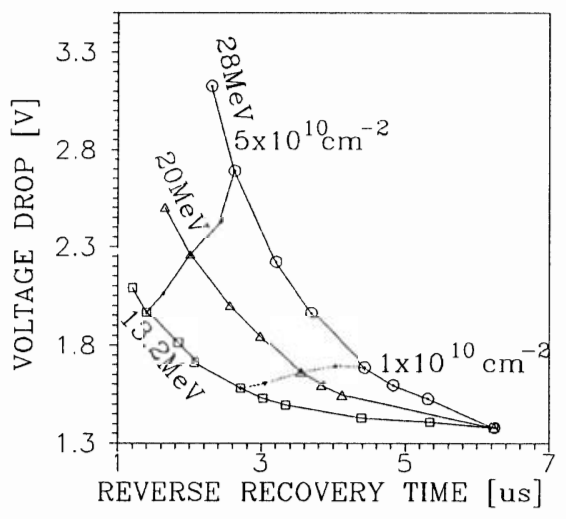

Fig.3: Forward voltage drop-reverse recovery time trade-off for helium (DUT from Figs. 1, 2, 4, 5) $\mathrm{t}_{\mathrm{rr}}:-20 \mathrm{~V} / 5 \Omega / 25 \mu \mathrm{H}$ from ON-state $\left(200 \mathrm{~A} . \mathrm{cm}^{-2}\right)$ 


\section{SIMULATION PROCESS FLOW CHART}
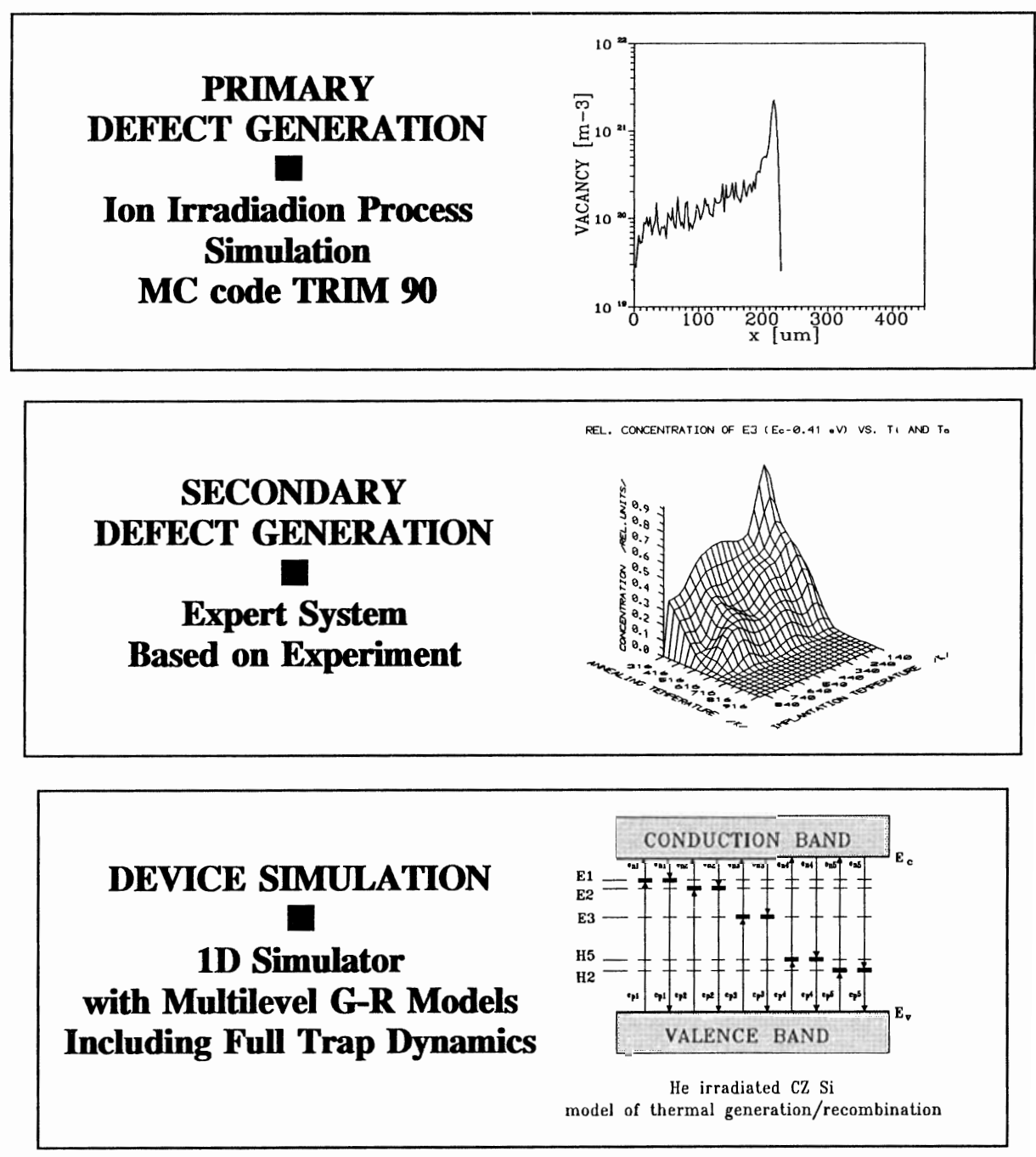

\section{SIMULATED DEVICE PERFORMANCE}

Reverse Recovery of a Power Diode

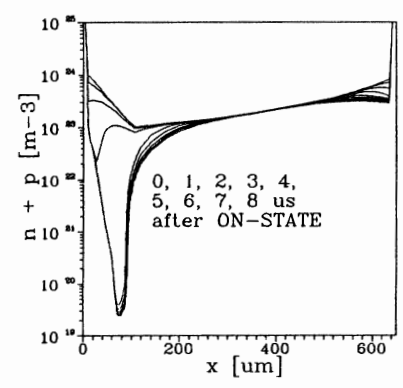


drop and diode reverse recovery time with the irradiation dose and energy as parameters. Figs. 4 and 5 provide a comparison of the new method (no.1) with the ordinary simulation based only on a simplestructured lifetime profile (no.2). For the case no.2, the maximum is approximated by rectangular lifetime profile of $\tau_{\mathrm{n} 0}$ and $\tau_{\mathrm{p} 0}$ magnitudes in SRH model, where FWHM values calculated by TRIM [5] are used as a width. In Fig.4, the curve no. 1 is in excellent qualitative agreement with measurements [3], while curve no. 2 exhibits unrealistic decrease of the the voltage drop for higher energies. In Fig.5, the curve no. 2 gives much more pesimistic trade-off between forward voltage drop and reverse recovery time in comparison with no.1. This is because both the curves no. 2 do not take into account the defects created along the whole ion track, i.e. before the concentration maximum.

\section{Conclusion}

For the first time, an accurate simulation of power devices that are subjected to high-energy low-dose ion irradiation has been presented and compared with standard simulation technique. The system proved useful for exact prediction of electrical parameters in conjunction with the irradiation technology.

This paper has been supported by the grant no. 8068 from CTU Prague.

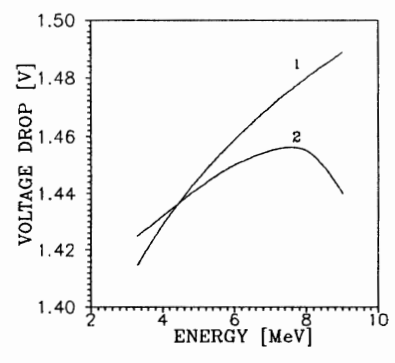

Fig.4: Forward voltage drop vs. irradiation energy of hydrogen (exact simulation curve no.1, simple-structured lifetime curve no.2) for the current density of 200 A. $\mathrm{cm}^{-2}$.

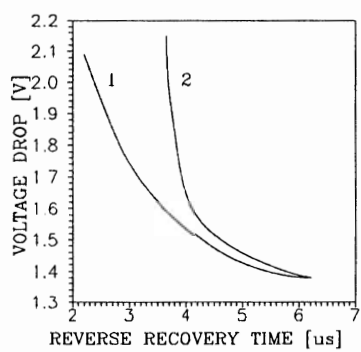

Fig. 5: Trade-off between forward voltage drop and reverse recovery time for hydrogen irradiation with energy of $5 \mathrm{MeV}$ (exact simulation no. 1, structured lifetime no.2).

\section{References:}

[1] V. A. K. Temple and F. W. Holroyd, IEEE Trans. Electron Devices, Vol. ED-30, no.7, pp.782 - 790, 1983

[2] M. Hátle, J. Vobecký, Proceedings of the MADEP'91, Florence, pp. 402 406, 1991

[3] A. Hallén, PhD. Thesis, Acta Universitatis Upsaliensis, Uppsala, Sweden, 1990

[4] P. Hazdra, V. Hašlar, M. Bartoš, Nuclear Instruments and Methods in Physics Research B55, pp. 637 - 641, 1991

[5] J. F. Ziegler, J. P. Biersack and U. Littmark, The Stopping and Range of Ions in Matter, Pergamon Press, New York, 1985

[6] A. Hallén, M. Bakowski, M. Lundqvist, Solid-State Electronics, Vol. 36, pp. $133-141,1993$

[7] P. Hazdra, J. Vobecký, Solid-State Electronics; submitted 